\title{
Elementos de la aduana de Manzanillo \\ COLIMA COMO FACILITADORES PARA LA INTEGRACIÓN DE SU PUERTO A LA CADENA DE SUMINISTRO GLOBAL
}

\section{Oscar Bernardo Reyes Real}

\section{Ariel Gutiérrez Ortiz}

\section{Carlos Méndez González ${ }^{1}$}

\section{Resumen}

La aduana es un elemento que resulta indispensable para concretar las operaciones de comercio exterior (despacho de mercancías de importaciones y exportaciones), siendo sus principales facultades la fiscalización, recaudación y facilitación comercial. Por ello, el objetivo de esta investigación es identificar y correlacionar los elementos que la aduana de Manzanillo Colima tiene para facilitar la integración de su puerto a la cadena de suministro global, desde la perspectiva de las agencias aduanales. Derivado de lo anterior, se realizó esta investigación considerando elementos como: sistemas de seguridad, tecnologías de información, capacitación de personal y capacidad de respuesta de la aduana. La información se obtuvo a través de la aplicación de un cuestionario de 20 reactivos a una muestra de 97 agencias aduanales ubicadas en Manzanillo Colima. De los resultados encontrados se destaca que la capacitación de personal y las tecnologías de información están fuertemente relacionadas con la integración, mientras que los sistemas de seguridad y la capacidad de respuesta tienen una correlación moderadamente fuerte hacia la integración.

Palabras clave: aduana, integración, cadena de suministro global, puertos.

1 Facultad de Comercio Exterior de la Universidad de Colima. 


\section{Abstract}

The Customs Office is an element that is essential to realize the foreign trade operations (clearance of goods for import and export), and its main powers to supervise, monitor and control the entry and exit of goods. Therefore, the aim of this research is to identify and correlate the elements that Customs Office of Manzanillo, Colima has to facilitate the integration of its port to the global supply chain, from customs agencies perspective. Derived from the above, this research was conducted considering elements such as security systems, information technology, personnel training and responsiveness of Customs. The information was obtained through a questionnaire of 20 items to a survey of 97 customs agencies located in Manzanillo, Colima. The results highlighted that staff training and information technology are strongly related to integration, while security systems and responsiveness have a moderately strong correlation towards integration.

Keywords: customs, integration, global supply chain, seaports.

\section{Introducción}

El proceso de globalización requiere que los países luchen por alcanzar la optimización de sus procesos productivos, aprovechando eficazmente aquellas actividades en las que tienen ventajas comparativas. En este contexto, para que las economías sean más competitivas en el escenario internacional necesitan mejorar los costos, la variedad, la disponibilidad y la facilitación de los flujos de bienes, dado que ello opera como una condición fundamental para el desarrollo y consolidación de las economías. Con el objetivo de cumplir con tal condición, los agentes de una economía tendrán que comprometerse para promover y consolidar los procesos de producción y comercialización; y es aquí en donde el transporte y la cadena de suministro se constituyen en un instrumento indispensable para el logro integral de dicho objetivo.

El papel que llevan a cabo los puertos dentro de la cadena de suministro es, sin duda, sumamente estratégico, debido a que tienen la capacidad de influenciar de manera significativa el costo final de un 
producto. Si se alcanza un mayor nivel de eficiencia en un puerto, no sólo desde la perspectiva de los servicios al buque sino también desde su integración al sistema de la cadena, implementación de tecnología informática y conectividad ferroviaria, vial y fluvial, se pueden reducir significativamente los costos del transporte interno, aumentar la competitividad y fomentar el comercio internacional.

Los puertos son la puerta principal del comercio internacional y operan como nodos centrales de la red física del transporte marítimo, movilizando las mayores cargas a nivel global, constituyendo además una interfaz entre modos de transporte, tanto para los trayectos nacionales como internacionales. Es por ello que, para mejorar la eficiencia en el movimiento de carga comercial, los puertos deben ser parte integral de la cadena de suministro (producción, transporte y distribución). Es decir, tener en cuenta no sólo las actividades que se efectúan en el marco del ámbito portuario, sino también la influencia que sus actividades tienen sobre el transporte anterior y posterior al puerto (Tomassian, Pérez y Sánchez, 2010).

Para Jiménez y Hernández (2002) La cadena de suministro se define como el conjunto de empresas integradas por proveedores, fabricantes, distribuidores y vendedores (mayoristas o detallistas) coordinados eficientemente por medio de relaciones de colaboración para colocar los requerimientos de insumos o productos en cada eslabón de la cadena en el tiempo preciso al menor costo, buscando el mayor impacto en las cadena de valor de los integrantes con el propósito de satisfacer los requerimientos de los consumidores finales. El adecuado desempeño de la cadena de suministro depende en gran parte del transporte.

El puerto de Manzanillo es considerado como el puerto número uno a nivel nacional en cuanto al movimiento de carga contenerizada, lo que ha exigido que cada uno de los actores del comercio exterior desempeñe sus actividades en condiciones de máxima calidad y debido a estas exigencias ha surgido la necesidad de cooperación entre estos actores para agilizar procesos y seguir en la competencia global (API, 2014). 
A pesar de ser el puerto del pacífico con mayor alcance territorial hacia el interior del país (hinterland), Manzanillo presenta algunos problemas de integración modal y de enlaces terrestres que, de no ser mejorados, pueden restarle competitividad, dificultando su consolidación como nodo de integración de cadenas productivas globalizadas y como posible puerto de tercera generación del pacífico mexicano, según lo indica el Programa Maestro de Desarrollo Portuario 2012-2017.

En 2017, el puerto de Manzanillo incrementó el manejo de carga contenerizada en $47 \%$ con respecto al 2012, movilizando 2 millones 830 mil 370 TEU (contenedor de 20 pies) en comparación con el millón 930 mil 893 operados durante el 2012. Se prevé que en los próximos años el crecimiento sea sostenido (API, 2018).

Lo anterior, sin duda, representa un enorme reto para las autoridades y operadores portuarios, ya que su competitividad futura depende de la productividad de los modos de transporte que le proveen carga, razón por la cual es importante analizar la cadena de suministro completa para lograr una complementariedad entre modos de transporte (Pérez, 2009).

Dado lo antes descrito, la presente investigación tiene por objetivo identificar y correlacionar los elementos que la aduana de Manzanillo Colima tiene para facilitar la integración de su puerto a la cadena de suministro global, desde la perspectiva de las agencias aduanales. Asimismo, esta investigación tiene repercusión práctica sobre la actividad empresarial, el desarrollo del puerto y la función de éste en la cadena de suministro global aportando información valiosa que servirá de material de reflexión y acción sobre el servicio de los puertos de contenedores mexicanos. Se compone de la siguiente manera: se realiza un análisis de la literatura, seguido de la descripción de la metodología empleada, posteriormente se muestran los resultados obtenidos y finalmente se presentan las conclusiones del mismo.

\section{Cadenas de suministro y su integración}

A medida que va pasando el tiempo las actividades relacionadas con el comercio internacional van creciendo y, por consecuencia, se incre- 
mentan las transacciones comerciales entre diversos países. Varios de ellos se encuentran inmersos en bloques comerciales. Para llevar a cabo las actividades propias del comercio internacional participan diferentes compañías que hacen posible el movimiento de mercancías formando una cadena en la cual todas las empresas están conectadas entre sí, lo que contribuye a que los bienes lleguen en tiempo y forma a los clientes/consumidores.

Para Beamon (1998) la cadena de suministro implica un proceso de fabricación estructurado donde la materia prima se transforma en bienes terminados para luego ser entregados a los clientes. Por otro lado según Chow y Heaver (1999), se puede decir que una cadena de suministro es el grupo de fabricantes, proveedores, distribuidores, detallistas, transportistas, información y otros proveedores de servicio de administración logística que están comprometidos en proporcionar bienes a consumidores. Ayers (2001) señala que la cadena de suministro es considerada como la integración de bienes físicos, información y flujos financieros, cuyo objetivo es satisfacer al consumidor final que requiere bienes y servicios de proveedores diversos. Finalmente, el autor Pienaar (2009), citado por Assey (2012), define la cadena de suministro como una descripción general de la integración de procesos, que implica organizaciones para transformar materiales crudos a bienes acabados y su transportación al usuario final.

Derivado de la globalización, cada vez es más relevante la interdependencia entre productores y mayoristas en las cadenas de suministro internacionales (Fourie, 2006). La cadena de suministro requiere de una coordinación estratégica entre las organizaciones implicadas en los diversos procesos y actividades que generan valor en bienes y servicios del productor hasta el cliente final (Christopher,1998). En relacion a lo anterior, Para Grant, Lambert, Stock y Ellram (2006), la administración de la cadena de suministro se refiere a la integración de procesos empresariales con valor agregado desde el usuario final hasta los proveedores. En tanto que, para Panayides (2006), la integración proporciona agilidad a lo largo de la cadena de suministro. 
Las cadenas de suministro generan fuertes obligaciones entre los participantes de la estructura de la cadena con el fin de lograr ventajas competitivas en algún diseño explícito. Tal condición permite observar la necesidad de establecer vínculos más estrechos entre las unidades productivas participantes que las obliga a mantener interacciones sistemáticamente (Durango, 2008).

Los puertos son un elemento fundamental en la cadena de suministro, es considerado como parte de un grupo de organizaciones en qué la logística y diferentes operadores de transporte están implicados en generar valor a los consumidores finales. Para lograr el éxito es necesario conseguir un alto grado de coordinación y cooperación (De Souza, Geraldo, Beresford y Pettit, 2003). La competencia entre puertos, particularmente aquellos en el mismo rango geografico, se ha intensificado. Por lo que en la actualidad, la competitividad de un puerto depende en gran medida de su habilidad para integrarse en la cadenas de suministro globales. La identificación de los elementos que contribuyen a la integración del puerto/terminal a dichas cadenas sugiere la busqueda de estrategias con la finalidad de aumentar la integración y conseguir ventajas competitivas (Panayides y Song, 2009). El contexto de una cadena de suministro integrada se refiere a una colaboración entre varias firmas que generan un sistema de flujos y restricciones de recursos importantes. Lo anterior implica esfuerzos que permiten cumplir el compromiso con los clientes (Bowersox, Closs y Cooper, 2007). En los puertos marítimos convergen una gran cantidad de organizaciones especializadas en el manejo de diversos tipos de carga y dentro de los aspectos más relevantes es la búsqueda de la eficiencia a través de las economías de escala, lo que impacta directamente en la competitividad (Moreno, 2012).

Las autoridades portuarias juegan el papel de facilitadores, enfocándose en la provisión de superestructura e infraestructura para las operaciones de embarque, carga y descarga, almacenaje temporal y operaciones en el interior del puerto (Cullinane, Song y Gray, 2002; Tongzon y Heng, 2005).

Los puertos son fundamentales en la política económica de los países, ya que permiten hacer más eficiente el sistema de transporte de 
los mismos, fomentan el crecimiento del comercio con otros países, alivian la congestión de los principales corredores terrestres, mejoran los enlaces marítimos con las regiones insulares y periféricas de un país y refuerzan el transporte multimodal y la logística del transporte (Díaz-Bautista, 2008).

En la administración de la cadena de suministro (SCM, Supply Chain Management), el puerto tiene una posición estratégica en el sistema de la cadena de suministro global y lleva a cabo un papel muy activo. Esto se observa en los siguientes aspectos: primero, el puerto es el principio y el final de la transportación oceánica, el cual tiene el mayor porcentaje de transporte de carga. Por lo tanto, el puerto es el punto donde se reúne la mayor carga y se conecta con los otros modos de transporte. Cuando es necesario llevar a cabo actividades adicionales a las industriales, comercial o técnicas, el puerto tiende a lograr las mejores economías de escala; y segundo, el puerto es el mejor punto de combinación de los factores productivos (Wang, 2011).

La SCM se define como la coordinación sistemática y estratégica de las funciones tradicionales de las organizaciones con el propósito de mejorar su rendimiento a largo plazo de manera individual y colectiva (la cadena de suministro como un todo) (CLM, 2000). La definición reconoce la naturaleza estratégica de la coordinación entre los socios comerciales y explica el doble propósito de la administración de la cadena de suministro: mejorar la organización y hacer rendir la cadena de suministro (Song y Panayides, 2007).

La literatura observa que a mayor grado de integración a través de la cadena de suministro mejora el desempeño de una firma (Narasimhan y Jayaram, 1998; Johnson, 1999; Frohlich y Westbrook, 2001), esto mientras no haya proveedores y clientes desintegrados en términos de sus procesos de negocios (Armistead y Mapes, 1993; Frohlich y Westbrook, 2001) -todos ellos citados por Song y Panayides (2007)-. Los hallazgos, junto con la inherente naturaleza estratégica de coordinación, hacen que la integración de la cadena de suministro sea un concepto de gran importancia en la gestión de negocios (Song y Panayides, 2007). 
Vickery (et. al., 2003) enfatiza que las tecnologías de información integradas y las buenas prácticas fortalecen los vínculos entre compañías que ocupan diferentes posiciones dentro de la cadena de suministro (vínculos verticales como socios proveedores y relaciones más cercanas con los clientes; y vínculos horizontales como la formación de relaciones dentro de las empresas utilizando equipos de multifuncionales). El uso de las tecnologías de información integradas son un factor clave que ayuda a incrementar el flujo de información entre los participantes del proceso y con ello facilitar la integración de procesos que trasciendan las fronteras de la empresa (Bowersox y Daugherty, 1995; Lewis y Talalayevsky, 1997).

Las relaciones con los proveedores y clientes parecen ser centrales en el contexto de la integración de la cadena de suministro. La asociación de proveedores trata a éste como un colaborador estratégico (Narasimhan y Das, 1999). Las relaciones cercanas con el cliente lo involucran proactivamente, adquiriendo información de otros clientes acerca de sus necesidades de manera responsable (Song y Panayides, 2007).

De forma tradicional, las autoridades portuarias desempeñaron el papel de facilitadores comerciales enfocándose en la provisión de superestructura e infraestructura para las operaciones de los buques, carga/ descarga, almacenamiento temporal y operaciones dentro del puerto. Sobre esta base, la mayoría de la investigación en el área ha sido respecto a la eficiencia y rendimiento de los puertos o terminales de contenedores, un ejemplo se encuentra en los trabajos de Cullinane, Song y Gray (2002); y Tongzon y Heng (2005).

El trabajo académico sobre la integración de los puertos/terminales en las cadenas de suministro ha sido limitado. Probablemente el trabajo empírico más actualizado ha sido realizado por los autores Carbone y De Martino (2003), quienes adoptaron un caso de análisis para investigar la contribución del puerto de Le Havre en la creación de valor dentro de la cadena de suministro automotriz.

Desde 1994, los puertos mexicanos se especializan en carga contenerizada, desarrollan un departamento de manejo comercial, tratando 
de convertirse en plataformas logísticas y se organizan para desarrollar espacios de flujos. Tratan de incorporar a la carga alto valor agregado y priorizan el manejo tecnológico y el conocimiento buscando emular a los puertos del mundo desarrollado (Ojeda, 2008).

\section{El comercio internacional y el rol de la aduana}

Para los estados controlar sus fronteras es una función sumamente importante, toda vez que "el propósito de las fronteras es delinear la soberanía y el control legal, esencialmente permitiendo que las comunidades de personas divididas en naciones funcionen mientras mantienen el control sobre su propio territorio" (Bersin, 2012) citado por (Coyle, Cruthirds, Naranjo, \& Nobel, 2014).

Al haber definido el concepto de frontera, lo siguiente es definir cómo será controlada. Esta decisión -como lo sostienen (Coyle et al., 2014) - ha sido históricamente impulsada por el enfoque primario del país, para lo cual (Koslowski, 2011) y (Widdowson, 2007) citados por (Coyle et al., 2014) señalan que "el mecanismo de control ha caído generalmente en cuatro grandes categorías: control de los límites físicos de los ejércitos invasores; control de la migración de la población; el cobro de los derechos sobre los bienes internacionales que entran en su país; y el control de las mercancías que salen y entran en el país".

Entonces, se puede afirmar que a diario por las fronteras del mundo transitan un sin número de personas; se realizan miles de millones más de operaciones comerciales legales e ilegales, derivadas de las cuales se recaudan importantes montos de contribuciones al comercio exterior; se vigila y asegura el cumplimiento de diversas obligaciones en materia de barreras no $\operatorname{arancelarias}^{2}$ y se intercambian sumas considerables de

2 Las barreras no arancelarias o medidas de regulación o restricción no arancelaria como se conocen en México se entienden como "toda la gama de requisitos, reglamentos, medidas y otras formas administrativas que impiden o limitan el comercio exterior de un país diferentes a los aranceles" (Rohde, 2002: 280). Véase también como referencia legal en México el segundo párrafo del artículo 17 de la Ley de Comercio Exterior vigente en México, el cual establece que "estas medidas consistirán en permisos previos, cupos máximos, marcado de país de 
dinero que no son registradas en las cuentas públicas de las naciones, montos que regularmente son producto de transacciones realizadas con las organizaciones criminales internacionales, de ahí lo trascendente respecto de la determinación de cómo y quién ejercerá el control fronterizo.

Por lo expresado en los párrafos anteriores, la entidad pública a la que se le ha confiado el control de las fronteras, tradicionalmente ha sido reservada para las administraciones aduaneras o aduanas, en ese contexto, como lo refieren Cancino y Trejo (2015:98) "se ha considerado a la aduana como un punto de entrada y de salida de mercancías bajo el control del estado", es decir, el papel típico reconocido para las aduanas se había constreñido a una oficina vigilante y controladora del flujo comercial de bienes a lo largo de las fronteras de un país con el propósito principal de recolectar los derechos de aduanas.

No obstante lo referido en supra líneas, Cancino y Trejo (2015:98) expresan que la aduana tiene diferentes alcances, situaciones que estarán condicionadas al

enfoque y papel que juegan los agentes del exterior: Algunos la consideran como una autoridad; una institución; un grupo de personas facultadas; el lugar para importar o exportar mercancías; el puerto marítimo o fronterizo; el recinto fiscal o fiscalizado; el edificio donde se encuentra la oficina del director o administrador; la sala de entrada y salida de pasajeros internacionales; una garita; el conjunto de aduanas de todo el país, y hasta un servicio.

En otras palabras, la función de aduanera estará condicionada al entorno donde se le imagine y a su contexto cambiante.

Respecto al ambiente cambiante sobre el que la aduana ejerce sus facultades Gordhan (2007:56) señala que "a partir del dinámico y cambiante entorno en que operan las administraciones aduaneras, es importante que estas identifiquen y comprendan los principales impulsores estratégicos internacionales, regionales y nacionales para ser más re-

origen, certificaciones, cuotas compensatorias y los demás instrumentos que se consideren adecuados para los fines de esta Ley". 
ceptivos". Es decir, las aduanas deben de estar atentas a los cambios que el ambiente actual del comercio internacional está generando, con la finalidad de que su función se adapte a la nueva realidad en la que desarrollaran sus actividades y consecuentemente se alcancen los propósitos previstos de su misión.

Atinente a lo descrito en el párrafo anterior, el autor referido reconoce que los principales factores estratégicos que afectan a las administraciones aduaneras son:

el incremento del volumen del comercio internacional, el comercio liberalizado, nuevas normas internacionales, la proliferación de acuerdos comerciales regionales, el cambio significativo en los patrones tradicionales de comercio y el aumento en los participantes, la aparición de nuevos modelos de logística y cadena de suministros, el surgimiento de redes transnacionales de crimen organizado, las amenazas de seguridad que no reconocen fronteras, las preocupaciones cada vez mayores, con respecto a la salud pública y al medio ambiente (Gordhan, 2007:57).

Relacionado a lo descrito por Gordhan (2007), Shujie y Shilu (2009) coinciden respecto de que los factores externos clave que caracterizan el escenario del comercio global y el camino que deben de seguir las administraciones de aduanas para su adaptación a este nuevo entorno, siendo los siguientes los componentes: el incremento y diversidad del comercio entre naciones; patrones novedosos en el comercio y la logística, por ejemplo, la distribución just in time; la puesta en juego de la seguridad de las naciones por la escalada en las acciones del crimen organizado, la gran responsabilidad puesta en los hombros del sector público y su coordinación con el sector privado; los continuos cambios y pactos en las facilidades de intercambio.

Ahora bien, los factores expresados por Gordhan (2007) y Shujie y Shilu (2009) han modificado el contexto mundial de negocios, es decir, las barreras que antes eran un obstáculo para el intercambio de bienes tangibles entre los países, han ido desvaneciéndose en la medida en que la comunidad comercial internacional ha establecido nuevas reglas del 
juego. Las barreras arancelarias se han dejado de lado, privilegiando la rapidez en base a la atención de las necesidades de suministro de los actores del comercio internacional y el dinamismo del flujo comercial.

No obstante, este nuevo panorama del comercio internacional también ha favorecido a la delincuencia organizada, poniendo en un lugar privilegiado en los asuntos que atender de los países tópicos trascendentes tales como la seguridad de las fronteras, seguridad nacional, salud pública y medio ambiente, entre otros.

Por tanto, al tomar como punto de referencia los factores o impulsores estratégicos reconocidos por Gordhan (2007), impulsores que este mismo autor ha adjetivado como universales y que impactan a todas las administraciones aduaneras del orbe, se considera prudente mencionar que las afectaciones de éstos no han sido las mismas para todas las aduanas. Ya que la forma en que se le ha hecho frente es por cuenta de las diferentes administraciones aduaneras, que se han generado en diversos grados, partiendo de reformas o cambios integrales y hasta modificaciones de orden menor, como lo sostiene el autor citado.

Algunas de las tendencias de cambio identificadas, derivado de lo expuesto en el párrafo anterior y que refiere Gordhan (2007), son:

el reconocimiento por parte de la mayoría de las administraciones aduaneras respecto de que se ha producido un cambio fundamental en su función, la admisión de que la aduana es una parte central del proceso de globalización y un catalizador para la competitividad de los países y las empresas, la ampliación del papel de las Aduanas en la frontera, la distinción en relación a que el papel que desempeñan las aduanas es un rol importante en la administración del comercio internacional, el hecho de que la liberalización del comercio no significa que el papel de las Aduanas esté disminuyendo y finalmente el énfasis en la automatización, la gestión del riesgo y la inteligencia para facilitar el movimiento de los bienes legítimos, como elementos trascendentales para concentrar los recursos públicos en las operaciones comerciales de alto riesgo. 
Por otro lado, Widdowson (2007) señala que las modificaciones que hacen las aduanas en su papel, están directamente vinculados con la forma en la que organizacionalmente esté ubicada en la administración pública, es decir,

donde la recaudación de ingresos es el foco principal, la administración de aduanas generalmente forma parte de la cartera del Tesoro o de Finanzas. De manera similar, es probable que las administraciones que consideren que su papel es primordialmente de protección fronteriza estén alineadas con agencias que tienen un enfoque de gestión fronteriza (Widdowson, 2007:31).

En otros términos, el Ministerio, Agencia u Secretaría a la que se encuentre subordinada la aduana es un reflejo de los intereses de sus gobiernos.

Sobre lo señalado por Widdowson (2007) y en virtud de que México es una nación comercialmente abierta ${ }^{3}$, derivado de la extensa red de tratados y acuerdos comerciales celebrados con varios países, su participación en organizaciones internacionales importantes en la economía internacional y el intercambio comercial mundial como lo son la Organización Mundial del Comercio (OMC), la Organización Mundial de Aduanas (OMA) y la Organización para la Cooperación y el Desarrollo Económico (OCDE), resulta notable la situación de que la aduana en México este adherida a la Secretaria de Hacienda y Crédito Público $(\mathrm{SHCP})^{4}$, dependencia que, como parte de sus facultades primordiales, subordina la recaudación tributaria -basado en lo afirmado por Widdow-

3 México forma parte de 12 Tratados de Libre Comercio con 46 países, 32 Acuerdos para la Promoción y Protección Recíproca de las Inversiones (APPRIs) con 33 países y 9 acuerdos en el marco de la Asociación Latinoamericana de Integración (Secretaria de Economía, 2016).

4 Es la dependencia del Poder Ejecutivo Federal que tiene como misión proponer, dirigir y controlar la política económica del Gobierno Federal en materia financiera, fiscal, de gasto, de ingresos y deuda pública, con el propósito de consolidar un país con crecimiento económico de calidad (Secretaría de Hacienda Y Crédito Público, 2017). 
son (2007)-, supone que la finalidad imperante de la Aduana mexicana es la de recoger las contribuciones que genera su comercio exterior.

Por lo tanto, se puede afirmar que la forma en que actualmente se relacionan las economías de los países se ha modificado para dar lugar a un nuevo entorno del comercio, con nuevos retos y paradigmas. En el pasado, el intercambio comercial se limitaba al comercio de excedentes y el único objetivo de esos actos fue la cobertura de exigencias primarias de los ciudadanos que participaban en tal canje, pero la realidad actual es otra, las cantidades de bienes que transitan por el mundo se ha incrementado, las necesidades de los que participan en el comercio global han progresado teniendo como principal motor a nuevas formas de producir y realizar las transacciones ${ }^{5}$. Los cambios tecnológicos se presentan con una utilidad imprescindible en las operaciones del comercio internacional; las prioridades para los países que parten del comercio exterior se han transformado de la acción fiscalizadora con fines exactores a la acción facilitadora con una tendencia más hacia al proteccionismo de bienes jurídicos colectivos protegidos por las naciones como: la seguridad nacional, salud pública, sanidad animal y vegetal y ecología. Situación que, como ha sido referida por Gordhan (2007), Widdowson (2007) y Shujie y Shilu (2009), se identifica con aspectos determinantes de la función que deben tomar las aduanas en todos los países, hecho que hacia el interior de las fronteras también se verá afectada por los fines de política exterior buscados por el país al que pertenezca la aduana y que dará directriz a su papel político y operativo, como un eslabón más de la cadena de suministros.

\section{El papel de la aduana en la cadena de suministro}

La responsabilidad de las autoridades aduaneras varía de país en país y con frecuencia está sujeta a revisiones y modificaciones para asegurar su continua relevancia en un mercado de constante cambio. Sin embar-

5 Un ejemplo de las nuevas formas de hacer negocios se pueden percibir en el esquema productivo de just in time el cual se puede definir como una filosofía empresarial que se concentra en eliminar el desperdicio en todas las actividades internas o externas de la organización (Manufactura Inteligente, 2015) 
go, las aduanas han sido las responsables de implementar un amplio rango de políticas gubernamentales que les permitan obtener ingresos a través de la facilitación comercial de mercancías como: sustancias prohibidas, protección a bienes culturales, propiedad intelectual, etc.

Por varias décadas ha habido una presión de la comunidad comercial internacional para minimizar la intervención del gobierno en las transacciones comerciales y una gran expectativa de las autoridades aduaneras a nivel mundial para hacer un énfasis especial en la facilitación comercial. Esto se debe al ambiente de constante cambio en el cual las autoridades aduaneras operan; por ejemplo, la urgencia de tener aeronaves y buques más grandes, el comercio electrónico y la complejidad en los acuerdos comerciales. Las aduanas a nivel mundial han visto un incremento significativo en la carga laboral en todas las áreas impulsado por el marcado global y los avances tecnológicos.

Derivado del terrorismo, la seguridad en la cadena de suministro ahora tiene un pensamiento regulatorio, surge la Asociación Aduana-Comercio Contra el Terrorismo (C-TPAT, Customs-Trade Partnership Against Terrorism) y la Iniciativa de Seguridad de Contenedores (CSI, Customs Container Security Initiative), para proteger las cadenas de suministro globales de diversos ataques. Estas iniciativas han tenido un impacto mayor en la manera en que las aduanas y los demás miembros de la cadena de suministro llevan a cabo sus negocios. La idea del C-TPAT es proveer de protección en frontera y aduanas con un método de identificación y enfoque en los recursos de alto riesgo (Widdowson, 2007: 31-37).

Las organizaciones internacionales como la OCDE, el Banco Mundial, la OMA, la OMC y la Unión Europea consideran y recomiendan el uso de las tecnologías de información y comunicaciones (TIC) para intensificar la facilitación comercial y mejorar los procesos de las aduanas. La comunidad de negocios está buscando optimizar su situación financiera como una cuestión de absoluta necesidad durante las recesiones económicas. La adopción de las TIC junto con las mejores prácticas en las aduanas permite reducir costos sustancialmente. Las TIC son mediciones críticas estratégicas que ayudan a manejar las complejidades 
implícitas en el ambiente comercial global. El amplio uso de sistemas electrónicos en toda la cadena de suministro ha llevado a que los datos estén disponibles en un formato electrónico para el uso de las aduanas y otras agencias gubernamentales. Además de esto, el internet provee a las aduanas de una invaluable fuente de información para su control a lo largo de las fronteras, incluyendo la valuación y la clasificación de bienes y actividades de trazabilidad. La propagación de las TIC es una oportunidad para que las aduanas fortalezcan sus posiciones de vanguardia en el desarrollo estratégico de sus países (Lewis, 2009: 3-11).

Las organizaciones invierten en la capacitación, ya que es obligación de la empresa proveer a sus empleados de las herramientas necesarias para alcanzar la misión y las metas. En una organización como las aduanas, por su alto grado de responsabilidad y complejidad, es imperativo que los empleados sean capacitados sobre los fundamentos de las técnicas, prácticas, procedimientos y leyes que afectan el despacho de las mercancías. La calidad en las aduanas se puede definir como la precisión, uniformidad y certeza en sus acciones y decisiones. Esto sólo se alcanza mediante la capacitación del personal en conjunto con los sistemas y que la información se tenga en tiempo y forma. Las aduanas deben desarrollar un plan de capacitación, establecer un comité de la misma y crear una organización o academia para formación de empleados, con la finalidad de alcanzar sus metas. En vista del hecho de que las leyes y procedimientos que las aduanas ejecutan son en el campo internacional, las aduanas buscan una guía y apoyo de la OMA para asegurar precisión y uniformidad en la ejecución de sus actividades (Lane, 1998: 226).

En términos de despacho aduanero es importante considerar el ámbito de la gestión del riesgo, lo cual se debe incorporar a la cultura de la administración. Un sistema de gestión de riegos permite a la aduana manejar grandes volúmenes de cruces de frontera con escasos recursos. Asimismo, hace posible que la aduana mejore notablemente su desempeño en la facilitación del comercio, generando resultados positivos en cuanto a costo-beneficio, integración de las mercancías, los medios de transporte, los pasajeros y los sistemas de inteligencia; además de, mejorar 
la rapidez en las operaciones de carga y descarga y un incremento en la flexibilidad y capacidad de respuesta (Chávez, Orejel y Palacios, 2014).

Para el 2006 se delegó la responsabilidad de clasificar, determinar el valor en aduana y el origen de las mercancías a los agentes aduanales, quedando a cargo de la autoridad sólo la facultad de verificación del cumplimiento de esta obligación, así, se redujo la discrecionalidad de los empleados en la aduana. La instalación de equipo de alta tecnología ha permeado todas las áreas y procedimientos que se realizan, de tal forma que la red informática se actualiza en manera permanente y puede utilizarse en el ámbito nacional con reportes automatizados del quehacer aduanero. Además, los sistemas de control (videos, aforos y rayos X) son continuamente renovados (México, 2013).

Para la OMA, las aduanas promueven la prosperidad económica y el desarrollo social de los países. Las fronteras dividen, las aduanas conectan. Las acciones coinciden con la tendencia de automatizar todos los procesos para la logística a nivel global, atender los servicios derivados de un comercio internacional en ascenso y contribuir a que los procesos sean más ágiles, garantizando la seguridad en la cadena de suministro y la reducción del tiempo (Patiño, 2013).

\section{La Aduana de Manzanillo}

Los dos hitos más importantes que han impulsado a nivel mundial el incremento de la competitividad de las aduanas mediante el mejoramiento de procesos, infraestructura y el uso de tecnología de punta son el convenio de Kyoto y el Marco SAFE de la OMA. En México el Gobierno Federal y la Administración General de Aduanas establecieron como puntos de partida para el mejoramiento de la competitividad de las aduanas: el programa aduana modelo y el plan de modernización de las aduanas 2007-2012, no obstante, no ha sido lo único que el gobierno de México ha hecho con tal propósito, pero estos dos esquemas son los paradigmas en esta materia.

El programa aduana modelo según Reyes (2006: 32-33) tuvo como propósito mejorar la infraestructura, los procesos, la planilla de perso- 
nal y su interacción con los usuarios, todo lo anterior necesario para el óptimo funcionamiento de la aduana. Las aduanas que formaron parte de dicho proyecto fueron cuatro: Manzanillo, Veracruz, Nuevo Laredo y Reynoso, aunque en este estudio el énfasis se realizará en la aduana de Manzanillo.

En Manzanillo se mejoró la infraestructura con el proyecto aduana modelo, lo que permitió separar los flujos de vehículos particulares y de servicios al puesto de los transportes de carga, para evitar congestionamientos y agilizar su flujo. Se amplió el andén de primer reconocimiento, lo que incrementó la capacidad y calidad de servicio en dicho proceso. Se acondicionó el edificio de servicios múltiples del puerto como una medida temporal que permitió acercar las áreas administrativas de la aduana y se obtuvo un predio en el recinto fiscal del puerto donde se construyó un nuevo edificio administrativo. Se le dotó de cuatro equipos de inspección de transportes por rayos Gamma, un equipo de inspección de equipaje de pasajeros aéreos internacionales, dos patrullas equipadas con GPS (Sistema de posicionamiento global) y seis vehículos de uso rudo para el personal. Se seleccionaron y contrataron 34 servidores públicos adicionales a la plantilla original de 103 (Servicio de Administración Tributaria, 2006).

Con el plan de modernización de las aduanas 2007-2012 se le dio énfasis al mejoramiento de la infraestructura y la incorporación de tecnología de punta, a fin de lograr la efectividad de la gestión de la aduana, con lo que se buscó la facilitación del tránsito de mercancías y reducción de los costos de las operaciones de comercio exterior (Administración General de Aduanas, 2007). A este plan también fue incorporada la Aduana de Manzanillo. Con él se logró la competitividad de las aduanas en los aspectos tecnológicos, infraestructura, recursos humanos y organización administrativa, consiguiendo la agilización y facilitación de la operación aduanera, sin dejar de perder el control (Administración General de Aduanas, 2008).

Algunos de los resultados y proyectos de este plan fueron (Administración General de Aduanas, 2008): Incremento en el volumen de operaciones, incremento relativo de la recaudación debido a los tra- 
tados internacionales en materia comercial de los que México forma parte, fortalecimiento del capital humano; proyectos de simplificación: Modernización de sistemas, reingeniería de procesos, aduana sin papel, SAAI web, carriles FAST y Exprés, facilidades aduaneras, exportación simplificada, reanudación de actividades, alianza para el comercio seguro, homologación de criterios y ampliación de horarios; proyectos de control y combate al contrabando: esclusas tecnológicas, aforos, confinamiento de aduanas, phazir ${ }^{6}$, comunicación inalámbrica segura, administración de riesgos, combate a la piratería, video vigilancia administrada (VIVA), revasión en primer punto de entrada; proyectos de infraestructura: esclusas tecnológicas, confinamiento de aduanas, sistema de supervisión y control vehicular (Aforos), reordenamiento y ampliación de: Aduanas de Guadalajara; Lázaro Cárdenas; Aeropuerto de la Ciudad de México, Terminal 1 y 2; en Terminal 2, Proyecto de infraestructura y equipamiento de la Frontera Sur.

Actualmente en las Aduanas de México, incluyendo la de Manzani1lo, se están llevando a cabo dos grandes proyectos: el Modelo de Administración Tributaria de Comercio Exterior (MAT-CE) (Orantes, 2015) y el proyecto de Integración Tecnológica Aduanera (PITA), la finalidad de estos proyectos es la eficiencia en el manejo de la información de las operaciones de comercio exterior para la automatización, facilitación y agilización de los procesos de importación y exportación.

Por todo lo anterior la Aduana de Manzanillo es considerada una de las aduanas más modernas y competitivas de México, motivo por el cual fue elegida como caso para la realización de esta investigación.

\section{Metodología}

El enfoque utilizado en esta investigación es mixto y se trata de un diseño correlacional-descriptivo, ya que se identifican y describen las variables, y la relación que existe entre ellas, a través de mediciones estadísticas que permiten observar la medición del fenómeno, en este

6 Es un equipo portátil de escaneo de mercancías, especialmente de productos químicos o polvos, que emite un haz de luz infrarroja. 
caso identificar y correlacionar sí los Sistemas de Seguridad (SS), las Tecnologías de Información (TI), la Capacitación del Personal (RH) y la Capacidad de Respuesta (CR) de la aduana de Manzanillo son elementos que facilitan la integración de su puerto a la cadena de suministro global (INT). Asimismo, se realizó una prueba de hipótesis.

El universo de investigación lo componen las 130 agencias aduanales que para el 2014 eran miembros de las Asociación de Agentes Aduanales del Puerto de Manzanillo A. C. Debido a la amplia cantidad de sujetos de investigación y a la limitación en recursos se determinó una muestra haciendo uso de la fórmula de poblaciones finitas propuesta por Fischer, Navarro y Espejo (2012).

$$
\boldsymbol{n}=\frac{\sigma^{2} \mathrm{Npq}}{\mathrm{e}^{2}(\mathrm{~N}-1)+\sigma^{2} \mathrm{pq}}
$$

Donde:

$$
\begin{array}{ll}
\mathrm{n}=? & \mathrm{n}=\text { Muestra o número de encuestas } \\
\mathrm{N}=130 & \mathrm{~N}=\text { Tamaño de la población } \\
\sigma=1.96 & \sigma=\text { Coeficiente de nivel de confianza } \\
\mathrm{P}=50 \% & \mathrm{p}=\text { Probabilidad de éxito } \\
\mathrm{Q}=50 \% & \mathrm{q}=\text { Probabilidad de fracaso } \\
\mathrm{e}=5 \%(0.05) & \mathrm{e}=\text { Margen de error }
\end{array}
$$

Con respecto a la recopilación de los datos, el instrumento utilizado fue el cuestionario, el cual es el conjunto de preguntas preparadas cuidadosamente sobre hechos y aspectos que interesan en una investigación para su contestación por la población o su muestra (Zorrilla, 1993).

Aplicando la fórmula se obtiene una muestra de 97 agencias aduanales. A las cuales se les aplicó un cuestionario compuesto de 20 reactivos utilizando una escala tipo Likert, la cual, en sentido estricto, es una medición ordinal, que consiste en un conjunto de ítems presentados en forma de afirmaciones o juicios ante los cuales se solicita la reacción de 
los sujetos (Hernández, Fernández y Baptista, 2010). La composición del cuestionario es como sigue: de las preguntas 1 a la 5 , forman parte de la variable SS; de la 6 a la 11 son parte de la variable TI; de la 12 a la 16 pertenecen a la variable RH; y de la 17 a la 20 son parte de la variable CR. Las personas a las que se les aplicó la encuesta fueron los Ejecutivos de Tráfico, los cuales forman parte del personal de cada una de las 97 agencias aduanales derivadas de la muestra.

Con la finalidad de obtener la validez del instrumento, la cual es el grado en que éste mide aquello que pretende medir, y también conocer la fiabilidad en la consistencia interna del instrumento, es prudente estimar el alfa de Cronbach (Welch y Comer, 1988). Además, como lo establece Rivas (2009), citado por Valenzo, Galeana y Martínez (2015), se recomienda medir, siempre que sea posible, el alfa por variable, ya que esto permite conocer la consistencia del instrumento en general y la consistencia de cada variable en particular.

\section{Tabla 1. Prueba de confiabilidad por variable}

\begin{tabular}{|c|l|c|}
\hline No. & \multicolumn{1}{|c|}{ Variables independientes } & Alfa de Cronbach \\
\hline 1 & Sistemas de Seguridad (SS) & 0.831 \\
\hline 2 & Tecnologías de Información (TI) & 0.791 \\
\hline 3 & Capacitación del Personal (RH) & 0.852 \\
\hline 4 & Capacidad de Respuesta (CR) & 0.775 \\
\hline & Cuestionario completo & 0.871 \\
\hline
\end{tabular}

Fuente: elaboración propia con base en la investigación de campo y utilizando el SPSS v19.

Lo que se aprecia en la tabla 1 es la confiabilidad del instrumento que en total presenta una confiabilidad buena, mientras que por cada variable estudiada se nota que SS y RH son las que tienen una confiabilidad buena, TI y CR aceptable.

El análisis de las respuestas obtenidas de las agencias aduanales ubicadas en Manzanillo, Colima, México, indican el nivel de integración del puerto de Manzanillo a la cadena de suministro global desde la 
perspectiva de las variables independientes propuestas. Las 97 agencias aduanales que contestaron la encuesta. El rango de escalas para identificar el nivel de integración se presenta en la tabla 2, la cual se construyó con el uso de la puntuación máxima y mínima con el propósito de obtener rangos de escalas iguales. El procedimiento para calcular el valor máximo es el siguiente: se toma en cuenta que (5) es el máximo valor a obtener en la escala tipo Likert, posteriormente se multiplica por el número de ítems (20) dando como resultado 100. Para el valor mínimo se tiene que (1) es el valor mínimo en la escala tipo Likert y (20) el número de ítems, por lo que el resultado es de 20. Se prosigue a realizar la diferencia de valores (100-20) y se divide el resultado entre los 5 rangos de la escala tipo Likert, dando como resultado final rangos iguales de 16 puntos cada uno, enseguida se suma el valor mínimo y el rango y así se van estableciendo cada uno de los rangos a medir.

\section{Tabla 2. Rango de escalas para la investigación sobre el nivel de la Integración del puerto de Manzanillo, Colima a la cadena de suministro global}

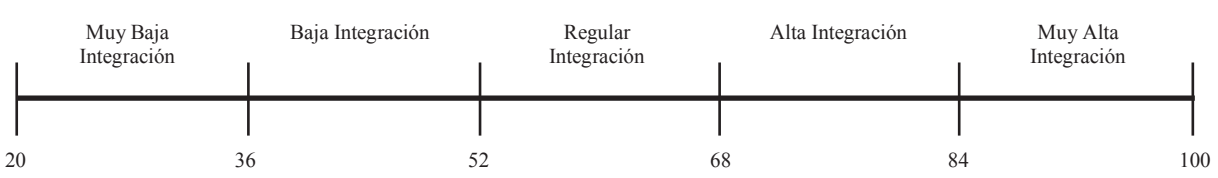

Fuente: elaboración propia con base en la investigación de campo.

Los datos obtenidos del trabajo de campo se procesaron en el programa SPSS versión 19 para Windows y en Microsoft Word y Excel. Con el uso del estadístico gamma se logró comprobar el grado de asociación de las variables independientes contra la variable dependiente, además se obtuvo el coeficiente de correlación de Spearman (Figura 3). 
Figura 3. Coeficiente de correlación de Spearman y el grado de asociación utilizando el estadístico Gamma y su incidencia en la variable Integración

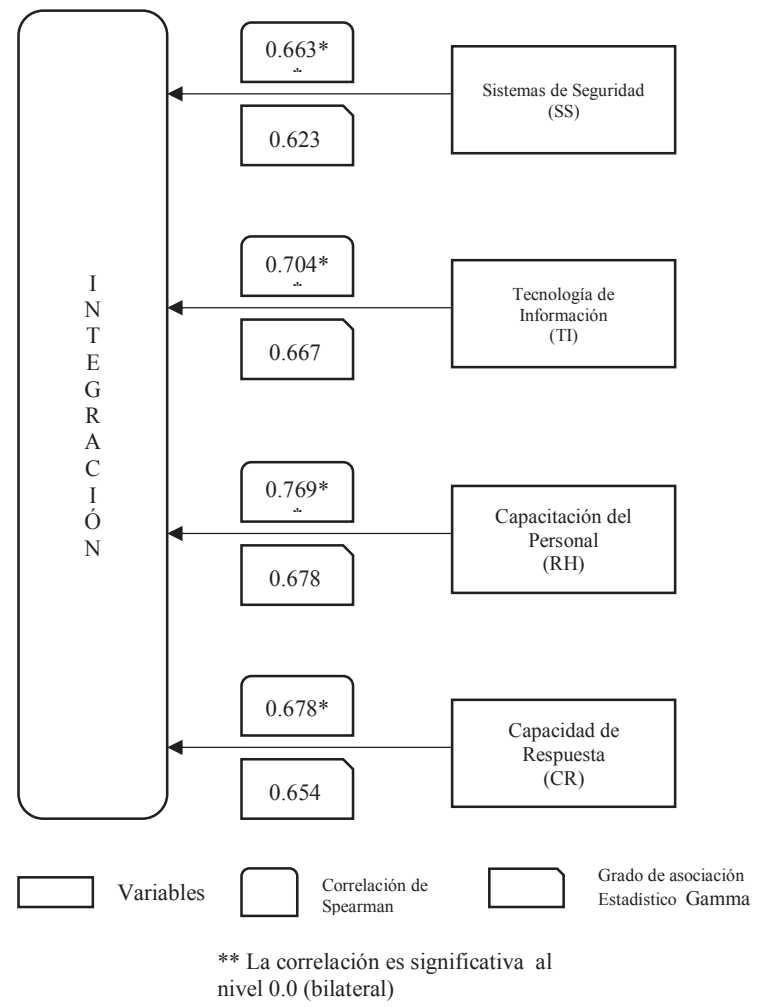

Fuente: elaboración propia en el trabajo de campo.

La figura 3 muestra los resultados que se encontraron en cada una de las variables independientes (SS, TI, RH, CR) y su correlación y grado de asociación con la variable dependiente Integración (INT). Para realizar este análisis se utilizaron las pruebas del tipo de datos ordinales como son: el coeficiente de correlación de Spearman y el grado de asociación del estadístico Gamma. Se aprecia que la variable RH tiene mayor correlación con la variable INT, de 0.769 , siendo una correlación moderada fuerte y con un grado de asociación de 0.678 ; seguida por la variable TI con una correlación moderada fuerte $(0.704)$ y un grado de 
asociación de 0.667 ; la variable CR presenta una correlación moderada fuerte de 0.678 y un grado de asociación de 0.654 ; y la variable SS tiene una correlación moderada fuerte de 0.663 con un grado de asociación de 0.623 .

\section{Discusión se resultados}

Con respecto a la variable SS, el $90 \%$ de los encuestados considera que es necesario tener una certificación en materia de seguridad, el $57 \%$ indica que las certificaciones más importantes son: C-TPAT, Nuevo Esquema de Empresas Certificadas (NEEC), Alianza Empresarial para el Comercio Seguro (BASC) y Seguridad en la Cadena de Suministro (ISO 28000). Por otro lado, el $60 \%$ comenta que es relativamente sencillo aplicar medidas para la protección de la información y documentación.

En la actualidad, para que una empresa funcione adecuadamente y pueda resolver sus problemas lo más pronto posible es necesario tener tecnologías de información, ya que éstas permiten que las actividades de la empresa estén integradas y que la comunicación sea efectiva. Por lo que, en relación a la variable TI el $71 \%$ de los encuestados comenta que las tecnologías de información que tiene la aduana son las adecuadas, mientras que el resto piensa que deberían existir más o aprovecharse de mejor manera. El 89\% comenta que el Sistema de Automatización Aduanera (SAAI) ayuda a agilizar las operaciones, mientras que el 85\% comenta que la Ventanilla Única de Comercio Exterior (VUCE), simplifica los flujos de información y ayuda a reducir tiempos y costos. El $53 \%$ indica que el Puerto Sin Papeles (PSP) le ha ayudado a facilitar las operaciones en el puerto, siendo importante señalar que este último fue desarrollado por la administración portuaria integral de Manzanillo.

Debido a que la capacitación de los recursos humanos es un elemento clave para mejorar su propio desempeño y el de la aduana, en la variable $\mathrm{RH}$, el $54 \%$ de los encuestados considera que la capacitación debe ser en el área de operación aduanera, mientras que el resto indica que debe ser en el área de trámites y asuntos legales. E1 54\% considera que la capacitación de los recursos humanos es constante, el $40 \%$ indi- 
ca que existe una frecuencia menor y el $6 \%$ revela que la capacitación es nula. De manera similar, los recursos humanos cuentan con habilidades y actitudes para desarrollar sus funciones, lo que permite generar un ambiente organizacional competitivo. Con respecto a las habilidades o actitudes que deben tener los empleados/funcionarios de la aduana son: $27 \%$ ética, $21 \%$ compromiso, $20 \%$ trabajo en equipo, $20 \%$ disponibilidad y $12 \%$ adaptabilidad.

Para resolver los distintos problemas que se presentan en las actividades de la empresa se debe tener una buena capacidad de respuesta. En la variable CR, el 55\% de los encuestados consideran que la aduana tiene una capacidad de respuesta adecuada, mientras que el 34\% indica que es poco adecuada y el $11 \%$ percibe que es inadecuada. Los factores que permiten tener una capacidad de respuesta adecuada, según los encuestados, son 34\% una combinación de experiencia laboral y grado de estudios, 33\% la experiencia laboral únicamente, 23\% una combinación del perfil del puesto y el grado de estudios y un 9\% sólo tener el perfil del puesto adecuado.

\section{Conclusiones}

Las organizaciones que pertenecen a una cadena de suministro presentan fuertes relaciones de interdependencia, en términos del cumplimiento de objetivos, de minimización de costos, aseguramiento de la calidad, entregas a tiempo y niveles de servicio.

En las opiniones que externan los encuestados, se pudo confirmar que la aduana es un eslabón que permite facilitar la integración del puerto de Manzanillo, Colima a la cadena de suministro global, esto en relación a diversos factores que influyen dentro de la misma aduana y que determinaron este análisis, tal como son: los sistemas de seguridad destacando el C-TPAT, el BASC, el NEEC y el ISO 28000. Con respecto a las tecnologías de información cabe resaltar el uso del SAAI, VUCE y PSP. En relación a la capacitación de personal, sobresale que, para las agencias aduanales, es de suma importancia la capacitación de los recursos humanos y que los perfiles del personal sean adecuados; mien- 
tras que la capacidad de respuesta de la aduana se aprecia que es buena y se destaca la importancia de la experiencia y el grado de estudios para incrementar dicha capacidad.

La atención de los factores mencionados en el párrafo anterior puede detonar el incremento en el manejo de carga contenerizada, el consecuente aumento en las operaciones de comercio exterior y la inminente derrama económica que esto implica.

El estudio de estas variables ha permitido conocer su comportamiento e incidencia para integrar al puerto de Manzanillo, Colima, México a la cadena de suministro global. En información proporcionada el sujeto de investigación, es decir las agencias aduanales, se ha conocido que 70 de los encuestados se encuentran en la escala de Alta Integración con 72.16 puntos. Por lo que, todas las variables propuestas inciden de manera positiva en la Integración, debido a ello el modelo propuesto se aprueba.

La contribución de este trabajo de investigación impacta directamente en la labor diaria que se lleva a cabo en el puerto de Manzanillo y muestra la situación que prevalece en el mismo, según la opinión de las agencias aduanales.

Con respecto a las limitaciones e investigaciones futuras, sería importante considerar el análisis de más variables bajo este mismo modelo, retroalimentado el cuestionario aplicado y viendo la posibilidad de replicarlo en otros puertos marítimos a nivel nacional, con la finalidad de conocer en qué medida los puertos mexicanos se integran a la cadena de suministro global.

\section{Bibliografía}

Administración General de Aduanas, Plan de modernización de las aduanas, 2007: http://www.aduanas.gob.mx/aduana_mexico/2008/ descargas/noticias/f_AvPlanMod.pdf, [consultado, 27 de Septiembre de 2015]. 
Avances del plan de modernización de aduanas 20072012: http://www.aduanas.gob.mx/aduana_mexico/2008/servicios/144_12137.html, [consultado, 27 de septiembre de 2015].

API, Programa Maestro de Desarrollo Portuario 2012-2017, 2014: http://www.puertomanzanillo.com.mx/esps/2110421/programa-maestro-de--desarrollo-portuario, [consultado, 22 de Marzo de 2014].

,Estadisticas historicas, 2018: http://www.puertomanzanillo. com.mx/esps/2110433/estadisticas-historicas, [consultado, 19 de Enero de 2018].

Armistead, C. G., y J. Mapes, "The impact of supply chain integration on operating performance", Logistics Information Management, 1993, pp. 9-14.

Assey, J. J., "A new introduction to supply chains and supply chain management: definitions and theories perspective", International Business Research, 2012, pp. 194-207.

Ayers, J. B, Handbook of supply chain management, Boca Raton: Taylor \& Francis Group, 2001.

Beamon, B. M, "Supply chain design and analysis: models and methods", International Journal of Production Economics, 1998, pp. 281-292.

Bersin, A., "Lines and Flows: The Beginning and End of Borders", Brooklyn Journal of International Law, 2012, pp. 389-406.

Bowersox, D. J., y Daugherty, P. J., "Logistics paradigms: the impact of information technologies", Journal of Business Logistics, 1995, pp. 65-80.

Bowersox, D. J., Closs D. J, y Bixb, M., Administracion y Logistica en la cadena de suministros, McGraw-Hill Interamericana, Mexico, 2007.

, Administracion y Logistica en la cadena de suministros, McGraw-Hill Interamericana, Mexico, 2007. 
Cancino, R., \& Trejo, P., Nuevo derecho aduanero electrónico, Liber iuris novum, S. de R. L. de C. V, México, 2015

Carbone, V., y De Martino, M., "The Changing Role of Ports in Supply Chain Management: An Empirical Analysis", Maritime Policy and Management, 2003, pp. 305-320.

Chávez, M., Orejel, A. y Palacios, S., México, una aduana de clase mundial, SAT-SHCP, Ciudad de México, 2014.

Chow , D., y Heaver, T., Logistics strategies for North America, Global Logistics and Distribution Planning: Strategies for Management, Donald Waters, The Institute of Logistics and Transport, London, 1999.

Christopher, M., Logistics \& supply chain management: strategies for reducting costs and improving services, Pitman Publishing, London, 1998.

CLM, What It's All About, Council of Logistics Management, Chicago, 2000.

Coyle, T., Cruthirds, K., Naranjo, S., y Nobel, K., "Analysis of current customs practices in the United States and a proposed model for world class Customs", World Customs Journal, no. 8, vol. 1, 2014, pp. 71-86.

Cullinane, K., Song, D. y Gray, R., "A stochastic frontier model of the efficiency of major container terminals in Asia: assessing the influence of admininstrative and ownership structures", Transportation Research, 2002, pp. 743-762.

De Souza, G., Beresford, A. y Petit, S.,"Liner shipping companies and terminal operators: internationalization or globalization", Maritime Economics \& Logistics, 2003, pp. 393-412.

Díaz-Bautista, A., "Los puertos en México y la política económica portuaria internacional", Observatorio de la Economía Latinoamericana, 2008, pp. 1-7. 
Durango, E., Integración de la cadena de suministro: alianza estratégica y ventaja competitiva para las pymes, Universidad Nacional de Colombia, 2008.

Fischer, L., Navarro, A. y Espejo, J., Investigación de mercados: un enfoque práctico, McGraw-Hill, México, 2012.

Fourie, Y., Structuring South African maritime supply chain (SC) for higher efficiency, PhD Dissertation, Johannesburg, 2006.

Frohlich, L. y Westbrook, R., "Arcs of Integration: An International Study of Supply Chain Strategies", Journal of Operations Management, 2001, pp. 185-200.

Gordhan, P., "Customs in the 21st century", World Customs Journal, 2007, pp. 49-54.

Grant, D., Lambert, D, Stock, J. y Ellram, L., Fundamentals of logistics manegement, McGraw-Hill, Berkshire, 2006.

Hernández, R., Fernández, C. y Baptista, P., Metodología de la investigación, McGraw-Hill, México, 2010.

Jiménez, E., y Hernández, S., "Marco conceptual de la cadena de suministro: un nuevo enfoque logístico", Publicación técnica 215 SCT, 2002, pp. 1-249.

Johnson, J., "Strategic integration in distribution channels: managing the interfirm relationship as a strategic asset", Jorunal of the Academy of Marketing, 1999, pp. 4-18.

Lane, M., Customs moderinization and the international trade superhighway, Quorum Books, London, 1998.

Lewis, G., "The impact of ICT on customs", World Customs Journal, 2009, pp. 3-11.

Lewis, I., y Talalayevsky, A., "Logistics and information technology: a coordination perspective", Journal of Business Logistics, 1997, pp. 141-157. 
Manufactura Inteligente, Just in time - JIT, 2015: http://www.manufacturainteligente.com/just-in-time-jit/, [consultado, 20 de abril de 2017].

Aduanas, Aduana México, 2013: http://www.aduanas.gob.mx/aduana_mexico/2008/quienes_somos/138_10004.html, [consultado, 15 de Noviembre de 2013].

Moreno, A., Análisis de los elementos que integran la cadena de suministro para sustentar la competitividad, Organización de Estados Americanos, Perú, 2012.

Narasimhan, R., y Das, A., "An empirical investigation of the contribution of strategic sourcing to manufacturing flexibilities and performance", Decision Sciences, 1999, pp. 683-718.

Narasimhan, R., y Jayaram, J., "Causal linkages in supply chain management: an exploratory study of North American manufacturing firms", Decision Sciences, 1998, pp. 579-605.

Ojeda, J., Cuatro puertos de México en un mundo globalizado: ¿entre la exclusión y el crecimiento (1982-2004)?, Tesis doctoral EUMED, Malaga, 2008.

Orantes, E. , MAT-CE, despacho QR, Presentado en Desayuno de gerentes INDEX Mexicali, Baja California, 2015: http://www. indexmexicali.org. $\mathrm{mx} / \mathrm{index} . \mathrm{php} /$ noticias/item/31-presenta-aduana-programa-mat-ce-de-comercio-exterior/31-presenta-aduana-programa-mat-ce-de-comercio-exterior, [consultado, 14 de Mayo de 2015].

Panayides , P., y Song, D., "Port integration in global supply chains: maesures and implications for maritime logistics", International Journal of Logistics Research, 2009, pp. 133-145.

Panayides, P., "Maritime logistics and global supply chains: towards a research agenda", Maritime Economics \& Logistics, 2006, pp. 3-18.

Patiño, D., "En el semáforo de Manzanillo", Revista T21, 2013, pp. 4649. 
Pérez, G., La necesidad de establecer politicas integrales de infraestructura, transporte y logística, CEPAL Boletín FAL, 2009, pp. 1-4.

Piennar, W., Introduction to Business Logistics, Oxford University, London, 2009.

Koslowski, R., The Evolution of Border Controls as a Mechanism to Prevent Illegal Immigration, 2011: http://www.migrationpolicy.org/ research/evolution-US-border-controls-illegal-immigration.

Rivas, T., Cómo hacer una tesis de maestría, La Salle, México, 2009.

Rohde, A., Derecho aduanero mexicano: Fundamentos y regulaciones de la actividad aduanera, Ediciones Fiscales ISEF, México, 2002.

Secretaria de Economía, México cuenta con 12 Tratados de Libre Comercio, 2016: http://www.gob.mx/se/articulos/mexico-cuenta-con-12-tratados-de-libre-comercio, [consultado, 20 de abril de 2017].

Secretaría de Hacienda y Crédito Público, Secretaría de Hacienda y Crédito Público | Gobierno | gob.mx., 2017: https://www.gob.mx/ shcp/que-hacemos, [consultado, 20 de abril de 2017].

Servicio de Administración Tributaria, Libro blanco 01, Aduana Modelo, 2006: ftp://ftp2.sat.gob.mx/asistencia_ftp/publicaciones/transparencia/Libros_Blancos/, [consultado, 13 de Noviembre de 2015].

Shujie, Z., y Shilu, Z., "The implication of customs modernization on export competitiveness in China", Reform by numbers, 2009, pp. 121-131.

Song, D., y Panayides, P., Global supply chain and port/terminal: integration and competitiveness, KNU Conference, Taiwan, 2007, pp. $1-14$.

Tomassian, G., Pérez, G., y Sánchez, R., Políticas integradas de infraestructura, transporte y logística: experiencias internacionales y propuestas iniciales, CEPAL, Recursos naturales e infraestructura, 2010, pp. 1-64. 
Tongzon, J., y Heng, W., "Port privatization, efficiency and competitiveness: some empirical evidence from coantainer ports (terminals)", Transportation Research, 2005, pp. 405-424.

Valenzo, M., Galeana, E. y Martínez, J., "Competitividad y supply chain management en la industria exportadora de aguacates en México", Revista Internacional Administración \& Finanzas, 2015, pp. 1-19.

Vickery, S., Jarayam,, J., Droge, C., y Calantone, R., "The effects of an integrative supply chain strategy on customer service and financial performance: analysis of direct vs indirect relationships", Journal of Operations Management, 2003, pp. 523-539.

Wang, L., "Study on port logisticsmarketing under the environment of supply chain", International Journal of Business and Management, 2011, pp. 267-271.

Welch, S., y Comer, J., Quantitative methods for public administration: techniques and applications, Brooks/Cole Pub. Co.,Virginia, 1988, p. 377.

Widdowson, D., "Changing role of Customs evolution or revolution?", World Customs Journal, 2007, pp. 31-37.

Zorrilla, S., Introducción a la metodología de la investigación, 2a ed., cal y arena, México, 1993, p. 391. 